\title{
The Effect of GABA Receptor on Information Capacity of Cultured Hippocampal Neurons
}

\author{
Geng Zhu ${ }^{1, *}$, Xiangning $\mathrm{Li}^{2}$, Hui Gong ${ }^{2}$, Yan $\mathrm{Zhu}^{1}$ and Xiaoou $\mathrm{Li}^{1}$ \\ ${ }^{1}$ Department of Biomedical Engineering, School of Medical Instrument, Shanghai University of Medicine \& Health Sciences, Shanghai \\ 201318, China \\ ${ }^{2}$ Britton Chance Center for Biomedical Photonics, Wuhan National Laboratory for Optoelectronics, Huazhong University of Science and \\ Technology, Wuhan, Hubei, 430074, China
}

\begin{abstract}
Neural information is encoded by action potentials delivered by neurons. Which component of neural activity constitutes the basic unit carrying information is still a controversial issue. In this paper, stimulation experiments using a network of hippocampal neurons cultured on a multi-electrode array are used to investigate this issue. The results show that for a set of pulse stimuli with varying voltage amplitude, the neuronal response to the fronto-potential sequence encodes more information through the moment of fronto-potential delivery than the number of fronto-potential deliveries, and that neurons at each locus are encoded independently of each other. After the addition of bicuculline inhibited the $\mathrm{GABA}_{\mathrm{A}}$ receptors, the information capacity decreased and the temporal resolution decreased, but the neurons at each site were still encoded independently. The results suggest that the encoding of stimulus amplitude in the cultured hippocampal neuronal network is better with spike timing than with count, and the effect of timing encoding is dependent on GABAA receptors.
\end{abstract}

\section{Introduction}

The use of information theory in neuroscience has opened the theoretical door to the study of neural coding. Various coding hypotheses have been tested in the visual, auditory, and olfactory nervous systems as well as in hippocampal neural networks by the informationtheoretic approach, e.g., the number of action potentials and the moment of action potential release have been shown to be related to the transmission and processing of information [1]. However, the network mechanisms of neural information processing are still unclear. In neuronal networks excitatory and inhibitory synapses regulate each other to make the network accomplish certain functions. When one of these synapses is affected, the function of the network will be altered. For example, GABAergic synapses are inhibitory synapses, and disruption of GABA transmission is often accompanied by epilepsy and schizophrenia [2]. In cultured neuronal networks, when GABAergic synapses are blocked, the usual phenomenon is a significant increase in both spontaneous and responsive firing rate. However it is not clear what is the role of GABAergic in neural coding and what is the significance for the neural network to achieve its function. So in this paper we will study this issue using an information theoretic approach.

The information-theoretic approach founded by Shannon in 1948 [3] is able to estimate the amount of information carried by a signal and can provide a method to achieve quantitative analysis of neural information.
Since Shannon's theory is based on the understanding of the system, while the brain is a black box relative to us, direct application of Shannon's theory in the study of the nervous system has encountered a number of problems. One of the key problems is how to make a correct estimate of the amount of information from experimental data. Direct estimation using Shannon's definition requires a large number of repeated experiments with as many as $2^{\mathrm{L} / \Delta \mathrm{T}}$ (typically $\mathrm{L}$ is $100 \mathrm{~ms}$ and $\Delta \mathrm{T}$ is $1 \mathrm{~ms}$ ) [4]. Information estimation methods have been continuously improved to apply to biological experimental data, and currently there are about three major types of information estimation methods (direct method, metric space method, binless method) [5]. Among them, the metric space method is able to estimate data with small sample size and is suitable for data with low distribution frequency and high temporal accuracy [6]. The results obtained using the information-theoretic approach is more general. In order to promote the use of information theoretic methods in the field of neuroscience, a series of toolkits of information theoretic methods have been developed and published in recent years. The information method used in this paper is the metric space method from the toolkit of [5].

Here, we compared the information capacity of the firing activity of normal and $\mathrm{GABA}_{\mathrm{A}}$ receptor inhibited hippocampal neuronal networks, to investigate the role of $\mathrm{GABA}_{\mathrm{A}}$ receptors in information processing.

\footnotetext{
* Corresponding author: zhug@sumhs.edu.cn
} 


\section{Materials and methods}

\subsection{Cell culture}

Wistar rats of 18 days gestational age (provided by the Experimental Animal Center of Tongji Medical College, Huazhong University of Science and Technology) were taken, anesthetized with ether, disinfected with 75\% alcohol and then removed from the fetal rats by dissection. Under aseptic conditions, the brain was quickly severed and the hippocampal tissue was isolated. Hippocampal tissues were digested with $0.125 \%$ trypsin at $37^{\circ} \mathrm{C}$ for $10 \mathrm{~min}$ and then terminated with inoculum (79\% DMEM, 10\% fetal bovine serum, 10\% horse serum, $1 \%$ glutamine). The cells were diluted to $\sim 1 \times 10^{6}$ cells $/ \mathrm{mL}$ and grown in $100 \mathrm{mg} / \mathrm{L}$ D-polylysine-treated MEA culture dishes. The electrodes and the surrounding $2 \mathrm{~mm}^{2}$ area were inoculated with $30 \mu \mathrm{l}$ of cell suspension, and $1 \mathrm{ml}$ of inoculum was replenished after $30 \mathrm{~min}$ and placed in a $37^{\circ} \mathrm{C}, 5 \% \mathrm{CO}_{2}$ incubator. $24 \mathrm{~h}$ later, the inoculum was completely replaced with growth solution (89\% DMEM, 10\% horse serum, 1\% glutamine), and the solution was changed every two and a half days thereafter. A layer of FEP film was covered on the MEA culture dish to maintain the osmotic pressure and $\mathrm{pH}$ of the culture medium, from which long time recordings and experiments were performed [7].

\subsection{Electrophysiological recordings and Stimulation experiment flow}

Electrical activities were recorded with a multi-channel system (MEA60 System, MCS GmbH, Germany), consisting of a square array of 60 substrate-embedded titanium nitride electrodes, $30 \mu \mathrm{m}$ in diameter and square spaced $200 \mu \mathrm{m}$ from each other, connected to an amplifier MEA1060; temperature controller; data collecting card and correlative software (MC_Rack). After the Petri dishes were removed from the incubator and placed on an amplifier connected to a temperature controller, the network spontaneous activity was observed by MC_Rack software (MCS GmbH, Germany).

The stimulation experiments were started after the frequency was kept stable (about $5 \mathrm{~min}$ ). Voltage stimulation was used. The stimulation waveform was bidirectional square wave pulse (single phase width of 200 $\mu \mathrm{s})$ with positive and then negative. In order to avoid mutual interference between stimuli, the interval between two pulses was $1 \mathrm{~s}$. The same stimulation sequence was given to the same electrode in the normal state and the bicuculline state. The stimulation sequence consisted of 160 pulses containing stimuli with voltage amplitudes of 150, 180, 200, 220, 250, 280, 300 and 400 $\mathrm{mV}$, every amplitude being repeated for 20 pulses. The response activity of the multipoint neurons in the network was recorded while the stimulation was performed.

\subsection{Entropy estimation method}

Calculating entropy by discrete histograms is called entropy estimation. In information theory, entropy is used to measure the lack of information in a system. Many kinds of entropy estimation methods exist, such as plugin (Plug-in), tpmc (Treves-Panzeri-Miller-Carlton) [8], jack (Jackknife) and nsb (Nemenman-Shafee-Bialek) [9]. Plugin is the classical estimation method, and is based on the definition of entropy.

$$
H=-\sum_{i} p_{i} \log _{2} p_{i}
$$

We use all these four methods to calculate the experimental data, and the results obtained do not significantly different. In this paper, we will use the plugin method for entropy estimation.

\subsection{Information theoretical analysis method}

Each trial was recorded containing the pulse stimulus and the signal for $100 \mathrm{~ms}$ after it. The spikes were extracted by a threshold detection method. The set of response spike sequences is constructed and the stimulus amplitude corresponding to the response is labeled, and each sequence corresponds to the response of one pulse stimulus.

In this paper, the ARMV metric defined by Aronov [10] is applied to compute the mutual information of multi-neuron responses and stimulus parameters. The ARMV distance is defined the minimum overhead required to transform a set of spike sequences into another. The overhead of adding or deleting a spike is 1 , the overhead of shifting displacement $\delta \mathrm{t}$ is $\mathrm{q}|\delta \mathrm{t}|$, and the overhead of relabeling a spike is $\mathrm{k}$. The distance between two sequences is calculated and clustered accordingly, and the mutual information of the set of sequences $\left\{X_{1}\right.$, $\left.\mathrm{X}_{2}, \ldots, \mathrm{X}_{\mathrm{m}}\right\}$ with the stimulus parameters is estimated according to the probability of correct clustering. If the distance between stimulus response sequences of the same magnitude is smaller than the distance between stimulus response sequences of different magnitudes, a good classification effect can be achieved. On the contrary, if the defined metric cannot distinguish between stimuli of different amplitudes, then the classification obtained is also random. For example, for our experimental data, the set of fronto-potential sequences of responses to 8 stimuli with different amplitudes, the information content $\mathrm{H}=3$ bits $\left(\log _{2} 8=\right.$ 3 ) for the most ideal case of classification.

The Redundancy index (RI) is used to quantitatively characterize the degree of independence of the neuronal response and is calculated by the following equation.

$$
\mathrm{RI}=\left(1-\frac{H_{j o \text { int }}}{H_{1}+H_{2}}\right) / 1-\frac{\max \left\{H_{1}, H_{2}\right\}}{H_{1}+H_{2}}
$$

where $H_{1}$ and $H_{2}$ are the amount of information carried by cell 1 and cell 2 alone, respectively, and $H_{\text {joint }}$ is the amount of information carried by taking cells 1 and 2 as a whole. For each value of $\mathrm{k}$ calculate the RI according to the above formula, where the q value is taken as the optimal value. When RI $=0, H_{\text {joint }}=H_{1}+H_{2}$, the two 
cells contribute independently to the mutual information; when RI $=1, H_{\text {joint }}=\max \left\{H_{1}, H_{2}\right\}$, the cell with less information does not contribute to the mutual information at all; when RI $>1$, the two cells carry contradictory information; when RI $<0$, the activities of the two cells are synergistic.

\section{Results}

Isolated hippocampal neurons cultured on multimicroelectrode dishes could establish synaptic connections after one week and form an extensively connected network between neurons after 3 weeks. Application of bidirectional voltage pulse stimulation to one electrode using a stimulator can cause stimulation artifacts and response firing activity to be recorded on other electrodes. For a health cultured neuronal network, the response activity of neurons has similar properties under the same stimulation conditions; while for different stimuli, different response activities are manifested (e.g., Figure 1a). By the response spike raster shown in Figure 1a, it can be seen that the average frequency of response spikes increases with increasing voltage amplitude and the moment of spikes issuing within 10-20 ms after stimulation is more fixed.

Here, we will use metric space method to analyze the amount of information carried by the activity of hippocampal neurons cultured on multi-microelectrode arrays in response to voltage stimuli of different amplitudes. The results showed the amount of information independently encoded by the two different channels varies with the temporal resolution (q) as shown in Figure $1 \mathrm{~b}$. When $\mathrm{q}=0$ is frequency encoding, the amount of information corresponding to it is smaller than that corresponding to $0<\mathrm{q}<1000$, indicating that temporal encoding is better than frequency encoding. As the value of $\mathrm{q}$ increases, the amount of information increases; the value of $q$ when the amount of information reaches its maximum is the optimal choice; after that, the increase of q corresponds to the decrease of the amount of information instead. This process suggests that for the optimal time-resolved (q) response spike firing moment is the most sensitive to the change in voltage amplitude. The optimal temporal resolution of cultured hippocampal neurons ranges from 200-1000 s-1.

When considering neuronal population coding, the analysis was performed using a multi-unit metric. The mesh formed by (q, k, $H_{\text {joint }}$ ), as shown in Figure $1 \mathrm{c}$, is the result of considering the joint encoding of amplitude information by neurons from two different loci. It can be seen that the value $H_{\text {joint }}$ is slightly greater than $H_{c h 75}$ and $H_{c h 22}$ and less than the sum of $H_{c h 75}$ and $H_{c h 22}$. the value of RI does not vary much with $\mathrm{k}$ and its value is slightly less than 1, implying that the contribution of the union of loci with little information to the encoding of information is small. It suggests that the information on stimulus voltage amplitude in the neuronal network is encoded independently by the neurons of each locus.

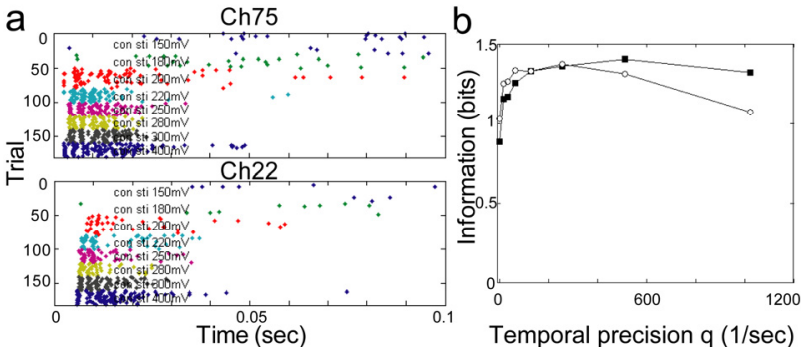

C

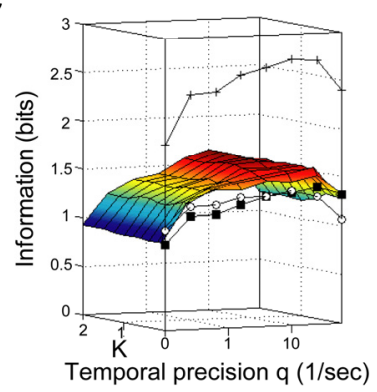

d

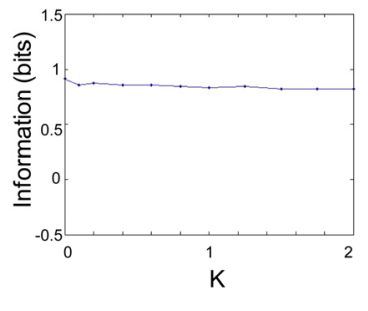

Fig1. Encoding of amplitude information by hippocampal neuronal networks

In the control state, a, the spike firing of two channels in the network for $100 \mathrm{~ms}$ after stimulation with different amplitude voltages is shown in the raster plot; b, the amount of information of these two dots as a function of temporal precision (q); c, the change of information of the two dots as a function of temporal precision $(\mathrm{q})(\mathrm{O}, \boldsymbol{\square})$ and the sum of both $(+)$, respectively, with the colored surface as a function of $H_{j o i n t}$ as a function of $\mathrm{q}$ and $\mathrm{k}$; $\mathrm{d}$, the $\mathrm{RI}$ as a function of $\mathrm{k}$ values

Inhibitory and excitatory transmitters play an important role in achieving the function of neural networks. Here, we added bicuculline (Bic) to the neuronal network to inhibit $\mathrm{GABA}_{\mathrm{A}}$ receptors and then gave the same stimulation sequence to the same electrodes and recorded the neuronal activities. From the spike raster graph, it can be seen that the response of neurons at each point in the network changed. Further analysis shows that the amount of information encoded independently for each site as a function of $\mathrm{q}$ value shows that the optimal temporal resolution becomes 50$200 \mathrm{~s}^{-1}$ and the corresponding amount of information decreases. $\mathrm{q}=0$ is still less than the amount of information corresponding to $0<\mathrm{q}<1000$, indicating that temporal encoding is still superior to frequency encoding. The results of the joint encoding of amplitude information by neurons at two different sites did not vary much, and the RI value still varied little with $\mathrm{k}$ and increased slightly compared to the normal state. This indicates that the stimulus voltage amplitude information is still encoded independently by neurons at each locus. The results suggest that the $\mathrm{GABA}_{\mathrm{A}}$ receptor does not affect the way of encoding, but can modulate the resolution of temporal encoding.

\section{Conclusions}

From the above results we can see that both frequency and temporal modalities encode certain information in the cultured hippocampal neuronal network. This shows 
that there may not be only one unique way of encoding in the central nervous system. In biological systems, it is possible that the noise present in the channel is overcome by the combined action of multiple encoding modalities to ensure the correct transmission of information.
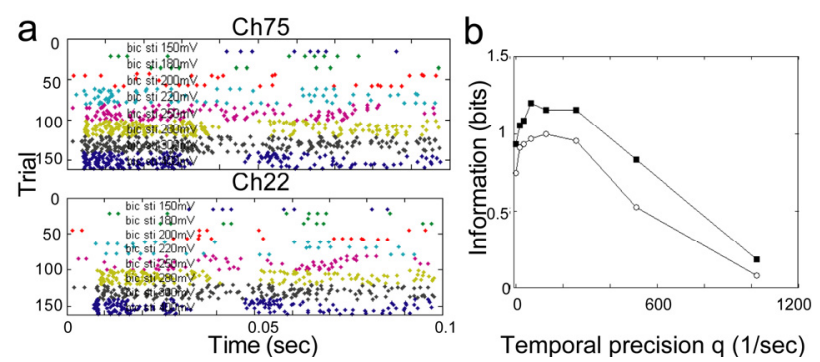

C

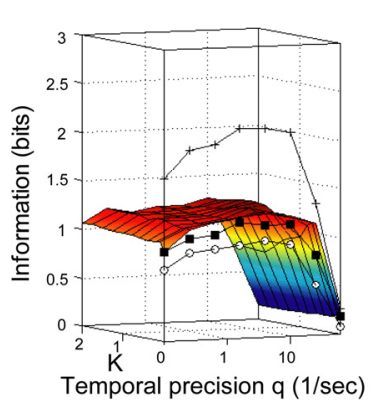

d

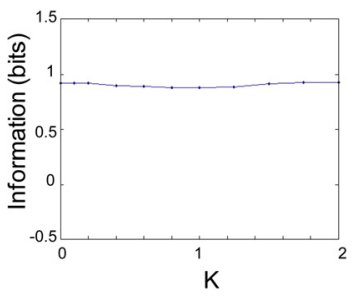

Fig2. Encoding of amplitude information by the hippocampal neuronal network with the addition of Bicuculline

After adding Bic, a, the spike firing of two channels in the network for $100 \mathrm{~ms}$ after stimulation with different amplitude voltages is shown in the raster plot; b, the amount of information of these two dots as a function of temporal precision (q); c, the change of information of the two dots as a function of temporal precision $(\mathrm{q})(\mathrm{O}, \square)$ and the sum of both $(+)$, respectively, with the colored surface as a function of $\mathrm{q}$ and $\mathrm{k}$ for $H_{\text {joint }}$; d, the function of RI as a function of $\mathrm{k}$ value

The results also show that the amount of information encoded in frequency is less than that encoded in time under normal conditions, but after the $\mathrm{GABA}_{\mathrm{A}}$ receptor is blocked the amount of information encoded in frequency is largely unaffected, while the amount of information encoded in time is greatly reduced. Thus, the presence of multiple encoding methods is beneficial for maintaining the stability of the system.

\section{Acknowledgments}

This work was supported by the National Science Foundation of China (Grant No. 31401147), Project funded by China Postdoctoral Science Foundation 2016M590358, 2020 Shanghai Municipal Education Commission Shanghai University Young Teacher Training Grant Program (Grant No. ZZSHJKYXY20007), 2021 Shanghai Health Medical College Teacher Talent 100 Pool Project.

\section{References}

1. VanRullen, R., R. Guyonneau, and S.J. Thorpe, Spike times make sense. Trends Neurosci, 2005. 28(1): 1-4.

2. Benes, F.M. and S. Berretta, GABAergic interneurons: implications for understanding schizophrenia and bipolar disorder. Neuropsychopharmacology, 2001. 25(1): 1-27.

3. C E, S. and W. Weaver, A mathematical theory of communication. Bell Syst. Tech. J, 1948. 27: 379423.

4. Strong, S.P., et al., Entropy and information in neural spike trains. Physical Review Letters, 1998. 80(1): 197-200.

5. Goldberg, D.H., et al., Spike train analysis toolkit: enabling wider application of information-theoretic techniques to neurophysiology. Neuroinformatics, 2009. 7(3): 165-78.

6. Victor, J.D., Spike train metrics. Curr Opin Neurobiol, 2005. 15(5): 585-92.

7. Zhu, G., et al., Transient alterations in slow oscillations of hippocampal networks by lowfrequency stimulations on multi-electrode arrays. Biomedical Microdevices, 2010. 12(1): 153-158.

8. Treves, A. and S. Panzeri, The upward bias in measures of information derived from limited data samples. Neural Computation, 1995. 7(2): 399-407.

9. Nemenman, I., F. Shafee, and W. Bialek, Entropy and Inference. Advances in Neural Information Processing Systems, 2002. 14.

10. Aronov, D., Fast algorithm for the metric-space analysis of simultaneous responses of multiple single neurons. Journal of Neuroscience Methods, 2003. 124(2): 175-179. 